\title{
High expression of cell adhesion molecule 2 unfavorably impacts survival in non-small cell lung cancer patients with brain metastases
}

\author{
Lu Dai ${ }^{1 \#}$, Yi-Hua Li ${ }^{2 \#}$, Ying-Ying Liang ${ }^{3}$, Jian Zhao ${ }^{1}$, Gang Chen ${ }^{4}$, Jun Yin ${ }^{1}$, Pieter E. Postmus ${ }^{5}$, \\ Alfredo Addeo ${ }^{6}$, Justin D. Blasberg ${ }^{7}$, Concetta Elisa Onesti ${ }^{8}$, Zhi-Wei Liao ${ }^{3}$, Xu-Guang Rao ${ }^{1}$, \\ Hui-Dong Long
}

${ }^{1}$ Department of Thoracic Surgery, Affiliated Cancer Hospital \& Institute of Guangzhou Medical University, Guangzhou, China; ${ }^{2}$ Affiliated Cancer Hospital \& Institute of Guangzhou Medical University, Guangzhou, China; ${ }^{3}$ Department of Radiation Oncology, Affiliated Cancer Hospital \& Institute of Guangzhou Medical University, Guangzhou, China; ${ }^{4}$ Department of Thoracic Surgery, Guangdong General Hospital, Guangdong Academy of Medical Sciences, Guangzhou, China; ${ }^{5}$ Department of Medical Oncology, Clatterbridge Cancer Centre, Liverpool Heart \& Chest Hospital, University of Liverpool, Liverpool, UK; ${ }^{6}$ Oncology Department, University Hospital Geneva, Geneva, Switzerland; ${ }^{7}$ Section of Thoracic Surgery, Department of Surgery, Yale School of Medicine, New Haven, CT, USA; ${ }^{8}$ Medical Oncology Unit, CHU Liège Sart Tilman and GIGA Research Center, Avenue de l'Hôpital 1, Liège, Belgium; 'Department of Medical Oncology, Affiliated Cancer Hospital \& Institute of Guangzhou Medical University, Guangzhou, China

Contributions: (I) Conception and design: HD Long; (II) Administrative support: YH Li; (III) Provision of study materials or patients: J Zhao; (IV) Collection and assembly of data: L Dai; (V) Data analysis and interpretation: XG Rao; (VI) Manuscript writing: All authors; (VII) Final approval of manuscript: All authors.

"These authors contributed equally to this work.

Correspondence to: Hui-Dong Long. Department of Medical Oncology, Affiliated Cancer Hospital \& Institute of Guangzhou Medical University, Guangzhou 510095, China. Email: longhuidong@126.com; Xu-Guang Rao. Department of Thoracic Surgery, Affiliated Cancer Hospital \& Institute of Guangzhou Medical University, Guangzhou 510095, China. Email: raoxuguanggyzl@163.com.

Background: Lung cancer is one kind of malignant tumor with a high risk for morbidity and mortality compared to other solid organ malignancies. Brain metastases occur in 30-55\% of non-small cell lung cancer (NSCLC) patients. Prognosis of NSCLC patients with brain metastases is very poor. Our previous study showed that cell adhesion molecule 2 (CADM2) could regulate the development of brain metastasis in NSCLC cells. Therefore, the objective of the study is to evaluate the effect of CADM2 on the prognosis of NSCLC patients with brain metastases.

Methods: The expression of CADM2 was detected by quantitative real-time polymerase chain reaction (qRT-PCR) in the tissue of the primary tumor. Patients were followed up and overall survival (OS) was calculated. The relationships between CADM2 and clinicopathological features were analyzed using the chisquare test. Kaplan-Meier analysis was carried out to demonstrate the influence of CADM2 on the OS of patients. Univariate and multivariate Cox analyses were used to determine the prognosis of NSCLC patients with brain metastases.

Results: A total of 139 NSCLC patients with brain metastases from the Affiliated Cancer Hospital \& Institute of Guangzhou Medical University, treated between January 2015 and December 2017 were evaluated retrospectively. The expression level of CADM2 in patients ranged from 1 to 17.2677 , with a median of 6.0772. Chi-square analysis showed that CADM2 gene expression level was not significantly associated with gender, age, tumor location, histological subtype, tumor $\mathrm{T}$ stage, extracranial metastasis, or smoking status. However, CADM2 expression was notably associated with risk for lymph node metastasis. The results of the Kaplan-Meier analysis showed that high expression [CADM2 messenger RNA (mRNA)

\footnotetext{
^ ORCID: 0000-0002-6895-9619.
} 
$\geq 6.0772]$ of CADM2 was markedly associated with poor prognosis. Univariate and multivariate Cox analyses demonstrated that CADM2 was an independent risk factor for survival in NSCLC patients with brain metastases $(\mathrm{P}<0.05)$.

Conclusions: CADM2 expression is up-regulated and closely associated with disease progression and poor prognosis in NSCLC patients with brain metastases. CADM2 expression warrants special consideration given its potential prognostic significance that might help inform clinical decision making.

Keywords: Non-small cell lung cancer (NSCLC); cell adhesion molecule 2 (CADM2); risk factor; prognosis; Cox analyses

Submitted Sep 27, 2020. Accepted for publication Apr 09, 2021.

doi: $10.21037 /$ jtd-21-307

View this article at: http://dx.doi.org/10.21037/jtd-21-307

\section{Introduction}

Lung cancer is the leading cause of cancer-related mortality throughout the world. According to Global Cancer Statistics 2018, lung cancer is the most commonly diagnosed caner (11.6\% of all cancer cases) and the leading cause of cancer death (18.4\% of all cancer deaths) (1). Non-small cell lung cancer (NSCLC) accounts for $85 \%$ of all lung cancer, with small cell lung cancer (SCLC) accounting for the remaining $15 \%$ (2). Clinically, approximately $80-90 \%$ of patients die as a consequence of local invasion and metastasis. Brain metastases represent a common complication in lung cancer patients, occurring in $30-40 \%$ of NSCLC and a significantly higher proportion of SCLC (3). Some NSCLC patients with brain metastases suffer from severe neurological impairment and experience a poor quality of life. The median survival time (MST) in this setting is approximately $1-3$ months (4).

Many studies have proven that clinical factors such as histology, stage, and age are rated to increased risk for developing brain metastasis in NSCLC patients (5-9). Waqar et al. $(10,11)$ shown that non-squamous histology, tumor size and grade, node-positive disease, and a younger age were relevant clinical factors for brain metastasis. In addition, a variety of biomarkers have also been reportedly associated with brain metastasis (12-16). Compared with epidermal growth factor receptor (EGFR) wild-type, patients with NSCLC harboring EGFR mutations have higher risk for brain metastases $(17,18)$. Moreover, brain metastases are more common in patients with a high expression of echinoderm microtubule-associated proteinlike 4 (EML4) and anaplastic lymphoma kinase (ALK) $(19,20)$.

Cell adhesion molecules (CADMs), also known as synaptic cell adhesion molecules (SynCAMs) or nectin-like molecules (Necls), consist of a protein family, the majority of which belong to the immunoglobulin superfamily. They are involved in the maintenance of cell adhesion, cell polarity and tumor suppression. CADMs, including CADM1, CADM2, CADM3, and CADM4, play an important role in tumor development, invasion and metastasis (21). In a variety of tumors, including prostate cancer, SCLC, and pancreatic cancer, the expression of CADM1 is downregulated (22). Furthermore, CADM3 and CADM4 have also been found to be down-regulated in prostate and colon cancers (23-25). CADM2, which is a member of the CADM family, is an immunoglobulin-like CADM. Our previous study showed that CADM2 could regulate the development of brain metastasis in NSCLC cells by inducing epithelial-mesenchymal transition (EMT) (26), suggesting that CADM2 may act as an oncogene and promote metastasis. However, the effect of CADM2 on the prognosis of NSCLC patients with brain metastases has not yet been reported. Based on our previous research, we hypothesize that CADM2 might affect the prognosis of NSCLC patients with brain metastases.

To confirm our hypothesis we performed a retrospective study in patients with NSCLC and brain metastases analyzing the relationship between overall survival (OS) and CADM2 expression. We present the following article in accordance with the REMARK reporting checklist (available at http://dx.doi.org/10.21037/jtd-21-307).

\section{Methods}

\section{Patients}

Patients from the Affiliated Cancer Hospital \& Institute 
Table 1 Reverse transcription-quantitative polymerase chain reaction primer sequences

\begin{tabular}{ll}
\hline Gene & Primer sequence \\
\hline CADM2 & F: 5'-GCTCTGGGCCTCATGGTTT-3' \\
& R: 5'-CAGCTGAGCAGAGGCAACTTT-3' \\
$\beta$-actin & F: 5'-GCATGGGTCAGAAGGATTCCT-3' \\
& R: 5'-TCGTCCCAGTTGGTGACGAT-3' \\
\hline
\end{tabular}

of Guangzhou Medical University treated between January 2015 and December 2017 were included in this retrospective study. We received approval from the local research ethics committee of Affiliated Cancer Hospital \& Institute of Guangzhou Medical University (No. 2020-57), and written informed consent was obtained from all subjects for sample collection and research use. All NSCLC diagnoses were cito-histologically confirmed and the presence of brain metastasis was radiologically confirmed. Tumor staging was assessed according to the tumor-nodemetastasis (TNM) staging system. Included cases fulfilled the following criteria: (I) patients with newly diagnosed NSCLC and brain metastases; (II) histologically or cytologically confirmed primary NSCLC, with no history of other tumor types; (III) brain metastases detected by cranial computed tomography (CT), cranial magnetic resonance imaging (MRI), or both; (IV) complete clinical characteristics profile data available (gender, age, tumor location, histology, T category, $\mathrm{N}$ category, extracranial lesions, smoking status) available; (V) availability of histological specimens; (VI) Chinese origin. All procedures performed in this study involving human participants were in accordance with the Declaration of Helsinki (as revised in 2013).

\section{Quantitative real-time polymerase chain reaction ( $q R T$ - PCR)}

Total ribonucleic acid (RNA) from lung tissue was isolated using TRIzol Reagent (Invitrogen, California, USA) according to the manufacturer's instructions. Next, the extracted RNA was reverse-transcribed into complementary deoxyribonucleic acid (cDNA) using the Prime Script RT reagent Kit (TaKaRa, Shiga, Japan). QRT-PCR was performed using Premix EX Taq ${ }^{\mathrm{TM}}$ (Probe qPCR) (TaKaRa), and the primer sequences are shown in Table 1 . The reaction conditions were as follows: pre-denaturation at $95^{\circ} \mathrm{C}$ for $3 \mathrm{~min}$, followed by 30 cycles at $95^{\circ} \mathrm{C}$ for $30 \mathrm{~s}$, annealing at $55^{\circ} \mathrm{C}$ for $30 \mathrm{~s}$ and extension at $55^{\circ} \mathrm{C}$ for $1 \mathrm{~min}$. qRT-PCR was performed using the ABI PRISM ${ }^{\circledR} 7300$ Sequence Detection System (Foster City, CA, USA). Each reaction was performed in triplicate. CADM2 expression was quantified by the $2^{-\Delta \Delta C t}$ method (13), and $\beta$-actin was used as an internal control.

\section{Statistical analysis}

Statistical analysis was performed using SPSS 22.0 software (SPSS Inc., Chicago, IL, USA). All data were expressed as mean \pm standard deviation (SD). Statistical differences were evaluated using Chi-square tests and Student's $t$-tests. Survival analysis was performed by means of Kaplan-Meier curves and log-rank test. The prognostic relevance of the clinicopathological data was analyzed using univariate and multivariate Cox regression analyses. $\mathrm{P}$ values of $\leq 0.05$ for two-sided tests were considered statistically significant.

\section{Results}

\section{Baseline characteristics of NSCLC patients with brain metastases}

One hundred and thirty-nine NSCLC patients with brain metastases were enrolled in this study. The baseline characteristics of the study group are summarized in Table 2 . There were 79 males and 60 females, with the mean age of 57 years (range, $31-78$ years). The mean duration of followup was 2 years.

\section{Follow-up}

Patients were followed up by outpatient consultations or telephone after enrollment. Considering that the team performing this study is different from medical oncologist treating the patients included, same date on treatment received are missing. Follow-up was continued until the patient died or was lost to follow-up, with a data cutoff 
Table 2 Baseline characteristics of NSCLC patients with brain metastases

\begin{tabular}{|c|c|}
\hline Variable & $\mathrm{N}$ \\
\hline \multicolumn{2}{|l|}{ Gender } \\
\hline Male & 79 \\
\hline Female & 60 \\
\hline \multicolumn{2}{|l|}{ Age (years) } \\
\hline$<65$ & 102 \\
\hline$\geq 65$ & 37 \\
\hline \multicolumn{2}{|l|}{ Tumor location } \\
\hline RUL & 36 \\
\hline RML & 8 \\
\hline RLL & 29 \\
\hline LUL & 36 \\
\hline LLL & 30 \\
\hline \multicolumn{2}{|l|}{ Histology } \\
\hline$A C$ & 116 \\
\hline NAC & 23 \\
\hline \multicolumn{2}{|l|}{ T category } \\
\hline $\mathrm{T} 1+\mathrm{T} 2$ & 49 \\
\hline $\mathrm{T} 3+\mathrm{T} 4$ & 90 \\
\hline \multicolumn{2}{|l|}{$\mathrm{N}$ category } \\
\hline $\mathrm{N} 1+\mathrm{N} 2+\mathrm{N} 3$ & 117 \\
\hline NO & 22 \\
\hline \multicolumn{2}{|c|}{ Extracranial lesions } \\
\hline Yes & 97 \\
\hline No & 42 \\
\hline \multicolumn{2}{|l|}{ Smoking } \\
\hline Yes & 62 \\
\hline No & 77 \\
\hline
\end{tabular}

NSCLC, non-small cell lung cancer; N, Number of patients; RUL, right upper lobe; RML, right middle lobe; RLL, right lower lobe; LUL, left upper lobe; LLL, left lower lobe; AC, adenocarcinoma; NAC, non-adenocarcinoma.

date of April 2020. The OS time was calculated from the date of pathological diagnosis until last available followup. Follow-up took place during two time periods: the first follow-up time was in April 2018; 68 patients had died, 65 patients were alive, and 6 patients were lost at follow-up.
The second follow-up time was in April 2020; 111 patients had died, 11 patients were alive, and 11 patients were lost to follow-up. In total, 17 patients were lost to follow-up (12.2\%). The overall 1-year survival rate was $72.7 \%$, overall 2-year survival rate was $37.4 \%$, overall 3 -year survival rate was $10.8 \%$, and the overall 4 -year survival rate was $5.0 \%$.

\section{Expression of CADM2 in NSCLC patients with brain metastasis}

CADM2 expression in the lung tissue of all 139 NSCLC patients with brain metastases was assessed by qRT-PCR. As shown in Figure 1, CADM2 expression ranged from 1 to 17.2677 , with a median of 6.0772 . Patients were divided into a high expression group (expression $\geq 6.0772$ ) and a low expression group (expression $<6.0772$ ) based on this median value.

\section{Relationships between CADM2 and the clinicopathological features of NSCLC patients with brain metastases}

Associations between CADM2 and clinicopathological features were analyzed by the chi-square test (Table 3). CADM2 expression level was not associated with gender, age, tumor location, histological subtype, tumor $\mathrm{T}$ stage, smoking status, or extracranial metastasis. A significant association between CADM2 expression and lymph node metastasis $(\mathrm{N})$ was observed $(90.1 \%$ of patients with CADM2 high were N-positive vs. $77.9 \%$ in the group CADM2 low, $\mathrm{P}=0.049)$. Patients with positive lymph nodes $(\mathrm{N} 1, \mathrm{~N} 2$, or N3) had a significantly higher level of CADM2 expression compared to those with negative lymph nodes (N0).

\section{High CADM2 expression is associated with poor prognosis}

Kaplan-Meier analysis demonstrated that patients with lower CADM2 levels had a longer OS compared to patients with increased levels of CADM2 [median OS: 23 vs. 17 months; $\mathrm{P}=0.021$; hazard ratio (HR): 1.49, 95\% CI: 1.013 to 2.191; Figure 2, Table 4].

\section{Univariate and multivariate Cox analyses for prognosis of NSCLC patients with brain metastases}

To identify potential prognostic significance, the univariate Cox model was applied to estimate individual clinical parameters for OS. The group with a value of 0 in Table 5 was considered as the control. No significant association 


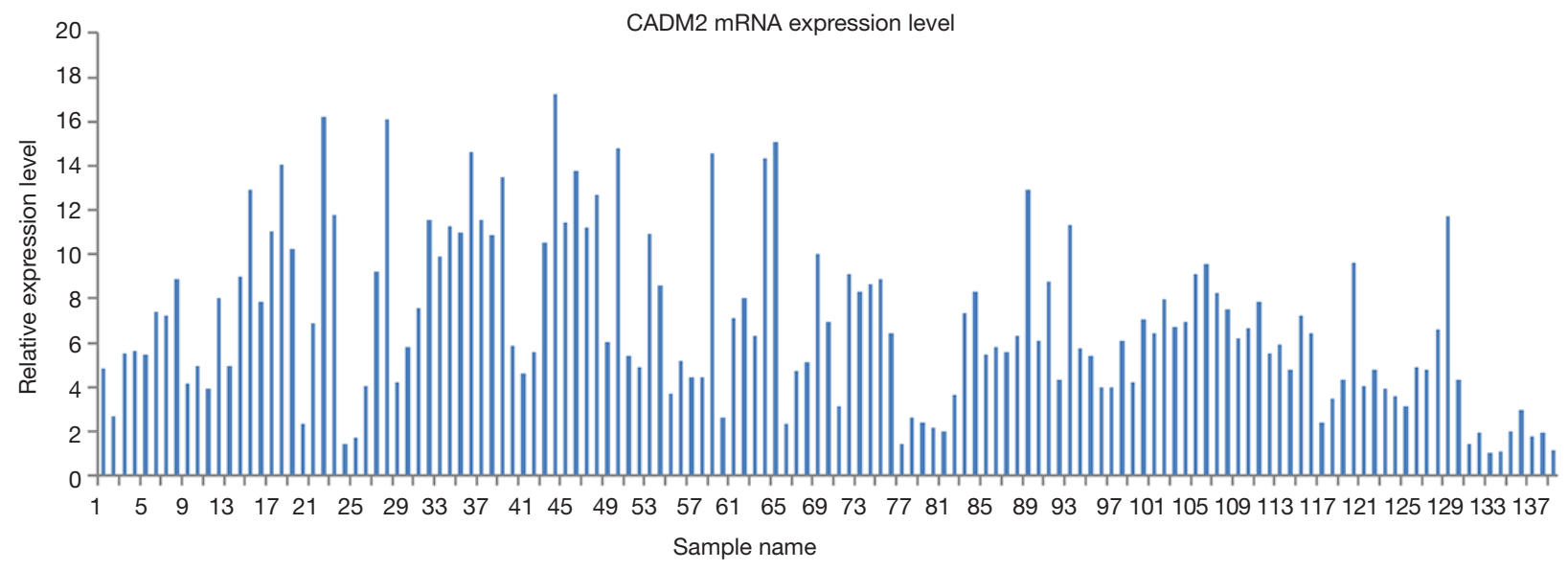

Figure 1 The expression of CADM2 in NSCLC patients with brain metastases. CADM2, cell adhesion molecule 2; NSCLC, non-small cell lung cancer.

was observed between OS and gender, tumor location, histological subtype, $\mathrm{T}$ stage, $\mathrm{N}$ stage, extracranial metastasis, or smoking status (Table 6). Conversely, age $(\mathrm{P}=0.031)$ and $\mathrm{CADM} 2$ expression level $(\mathrm{P}=0.026)$ were prognostic for OS in the univariate analysis (Table 6). These two variables were subsequently included in the multivariate Cox model, which showed that CADM2 expression level was an independent prognostic factor for OS, with a mortality rate in the high CADM2 group 1.49 times higher than that of low CADM2 group ( $\mathrm{P}=0.043$, Table 7).

\section{Discussion}

NSCLC patients with brain metastases are difficult to treat, due to the reduced distribution of cancer treatments across the blood brain barrier, a phenomenon which is associated with poor overall prognosis. Defining potential prognostic factors of brain metastases is of great clinical significance. Lee et al. (27) confirmed that pretreatment serum carcinoembryonic antigen (CEA) level was markedly related to brain metastases of advanced NSCLC. Our previous study demonstrated that CADM2 promoted brain metastasis by inducing EMT in human NSCLC (26).

CADM2 is expressed in many tissues, including liver, lung, and kidney. However, it is lowly expressed in tumors, especially in invasive and metastatic tumors. Li et al. (28) defined that overexpression of CADM2 could inhibit the EMT process, as well as the migratory and invasive ability of hepatocellular carcinoma (HCC) cells. They confirmed that CADM2 expression was significantly down-regulated in HCC tissues compared to normal according to The Cancer Genome Atlas (TCGA) data analysis and fresh HCC sample detection. Yang et al. (29) described that the expression of CADM2 was notably down-regulated in HCCs, and that expression was associated with serum alpha-fetoprotein (AFP), differentiation, vascular invasion, and hepatitis B surface antigen (HBsAg). In addition, they determined that CADM2 is an independent risk factor for HCC recurrence. Huang et al. (30) found that inhibition of microRNA (miR)-182 could suppress cell viability, invasion, and angiogenesis in retinoblastoma through inactivation of the phosphoinositide 3-kinase (PI3K)/protein kinase $\mathrm{B}$ (AKT) pathway and CADM2 up-regulation. In a separate study, Liu et al. (31) demonstrated that CADM2 was markedly down-regulated in human glioma tissues compared with normal brain and glioma cell lines, and that CADM2 expression level was significantly decreased in high-grade glioma tissues. CADM2 also inhibited the migration and invasion of U87 and U251 cells. Lastly, He et al. $(32,33)$ reported that the CADM2 gene is downregulated in human clear renal cell carcinoma by DNA promoter hypermethylation and/or loss of heterozygosity. The loss of CADM2 expression was also associated with a higher tumor pathologic stage. The reason why CADM2 plays a different role in our study from prior reports may be that the background environment of the gene is different. Ultimately, the CADM2 mechanism is not clear and further study is needed.

Thus far, there have been no studies that have evaluated the relationship between CADM2 and prognosis in NSCLC 
Table 3 The relationship between CADM2 and the clinicopathological features of NSCLC patients with brain metastases

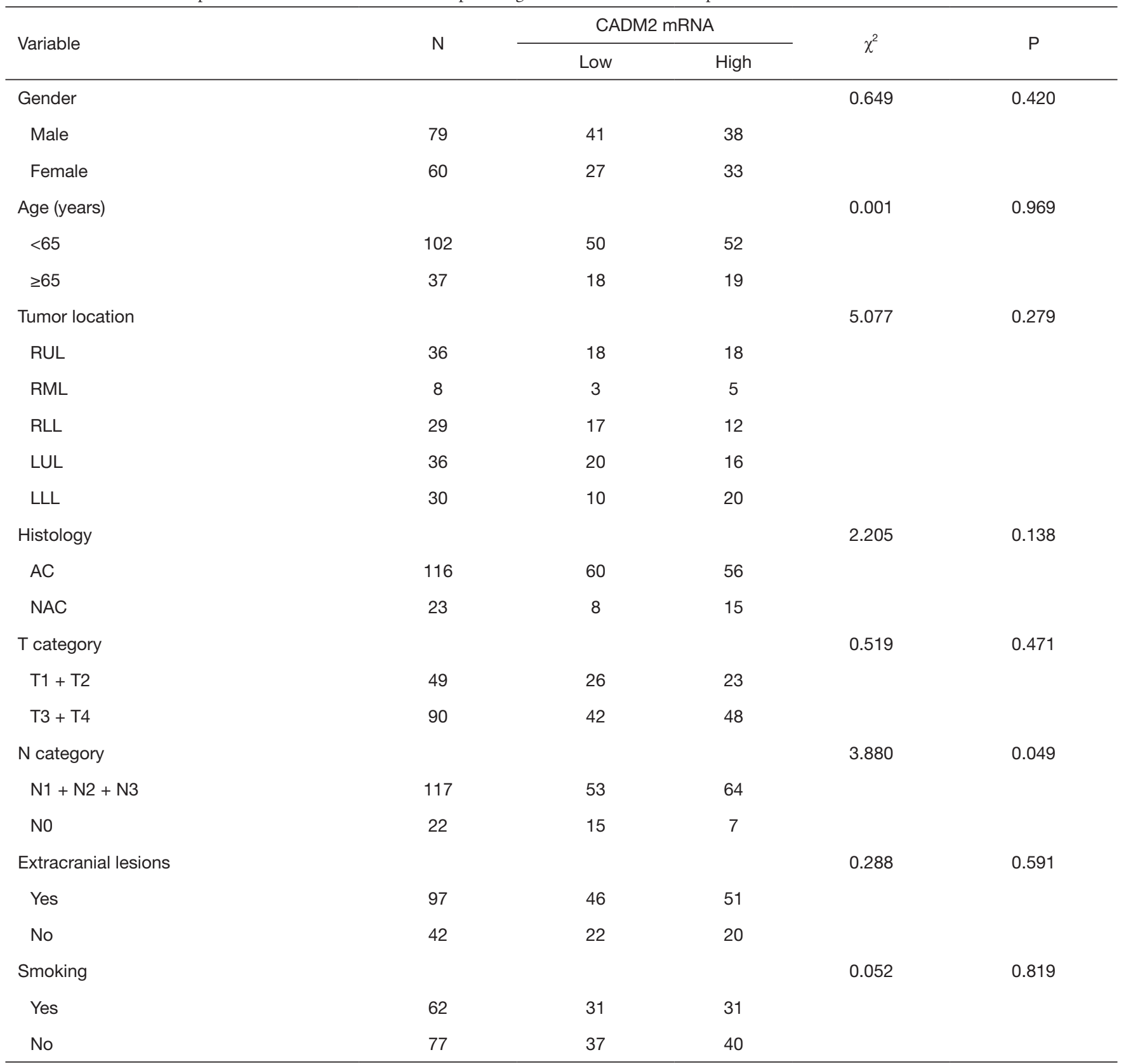

All statistical tests were two-sided and $\mathrm{P}<0.05$ was considered significant. NSCLC, non-small cell lung cancer; Low, CADM2 messenger RNA (mRNA) <6.0772; High, CADM2 mRNA $\geq 6.0772$; N, number of patients; RUL, right upper lobe; RML, right middle lobe; RL, right lower lobe; LUL, left upper lobe; LLL, left lower lobe; AC, adenocarcinoma; NAC, non-adenocarcinoma; CADM2, cell adhesion molecule 2.

patients with brain metastases. In our study, chi-square analysis identified that the level of CADM2 gene expression was not significantly correlated with gender, age, tumor location, histological subtype, tumor T stage, extracranial metastasis, or smoking. However, it was significantly associated with the presence of lymph node metastasis. Patients with positive lymph nodes had a markedly higher level of CADM2 expression. Our previous research showed that CADM2 was highly expressed in patients with brain metastases and CADM2 might regulate EMT (24). In this 


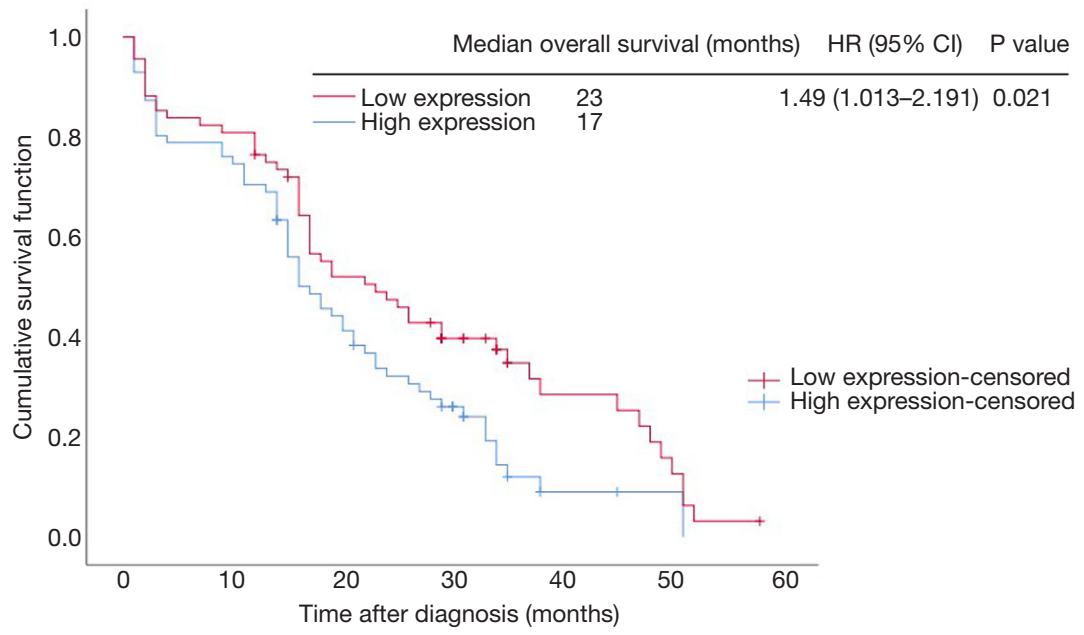

Figure 2 Kaplan-Meier analysis of the survival rate of NSCLC patients with brain metastases in relation to CADM2 expression. HR, hazard ratio; NSCLC, non-small cell lung cancer; CADM2, cell adhesion molecule 2.

Table 4 Kaplan-Meier survival analysis (log-rank test) according to the level of CADM2 in NSCLC patients with brain metastases

\begin{tabular}{|c|c|c|c|c|c|c|c|c|}
\hline \multirow{2}{*}{ CADM2 mRNA } & \multicolumn{4}{|c|}{ Mean OS (months) } & \multicolumn{4}{|c|}{ Median OS (months) } \\
\hline & Time & SE & $\mathrm{CL}$ & $\mathrm{CU}$ & Time & SE & $\mathrm{CL}$ & $\mathrm{CU}$ \\
\hline High & 20.127 & 1.796 & 16.607 & 23.646 & 17.000 & 2.033 & 13.015 & 20.985 \\
\hline Low & 26.610 & 2.234 & 22.230 & 30.990 & 23.000 & 4.013 & 15.135 & 30.865 \\
\hline
\end{tabular}

NSCLC, non-small cell lung cancer; OS, overall survival; Cl, confidence interval; SE, standard error; CL, lower confidence interval limit; CU, upper confidence interval; Low, CADM2 mRNA <6.0772; High, CADM2 mRNA 26.0772; CADM2, cell adhesion molecule 2.

Table 5 Cox regression analysis assignment table

\begin{tabular}{lc}
\hline Variable & Assignment \\
\hline Gender & Male $=1$, female $=0$ \\
Age (years) & $\geq 65=1,<65=0$ \\
Tumor location & $\mathrm{RUL}=1, \mathrm{RML}=2, \mathrm{RLL}=3, \mathrm{LUL}=4, \mathrm{LLL}=0$ \\
Histology & $\mathrm{AC}=1, \mathrm{NAC}=0$ \\
CADM2 mRNA & $\mathrm{High}=1, \mathrm{low}=0$ \\
T category & $\mathrm{T} 1+\mathrm{T} 2=1, \mathrm{~T} 3+\mathrm{T} 4=0$ \\
$\mathrm{~N}$ category & $\mathrm{N} 1, \mathrm{~N} 2, \mathrm{~N} 3=1, \mathrm{~N} 0=0$ \\
Extracranial lesions & $\mathrm{Yes}=1, \mathrm{No}=0$ \\
Smoking & Yes $=1, \mathrm{No}=0$ \\
\hline
\end{tabular}

The group with a value of 0 was considered as the control group. RUL, right upper lobe; RML, right middle lobe; RLL, right lower lobe; LUL, left upper lobe; LLL, left lower lobe; AC, adenocarcinoma; NAC, non-adenocarcinoma; Low, CADM2 mRNA <6.0772; High, CADM2 mRNA $\geq 6.0772$; CADM2, cell adhesion molecule 2 . study we found that CADM2 gene in NSCLC patients with brain metastases was associated with poor prognosis. Additionally, univariate and multivariate Cox analyses identified that CADM2 was an independent risk factor for poor survival of NSCLC patients with brain metastases. This implies that CADM2 plays an important role in brain metastases, and that expression may be a new target for prevention and/or treatment of NSCLC patients with brain metastases.

Nevertheless, this study has several limitations. Firstly, the number of patients is relatively small and should be expanded. Secondly, all patients were from a single hospital in China, which is not widely representative of NSCLC from around the world. Thirdly, considering that the team performing this study is different from medical oncologist treating the patients, data on anticancer treatment received by the patients included are missing. Thus, larger, highquality multicenter studies are needed for further validated our finding. 
Table 6 Univariate survival analyses (log-rank test) according to the clinicopathological features in NSCLC patients with brain metastases

\begin{tabular}{|c|c|c|c|c|c|c|}
\hline Variable & B & SE & Walds & $\mathrm{P}$ & $\mathrm{HR}$ & $95 \% \mathrm{Cl}$ \\
\hline Age (years) & -0.472 & 0.218 & 4.672 & 0.031 & 0.624 & $0.407-0.957$ \\
\hline Tumor location & & & 0.941 & 0.919 & & \\
\hline RUL & -0.107 & 0.273 & 0.154 & 0.695 & 0.898 & $0.526-1.535$ \\
\hline RLL & -0.084 & 0.287 & 0.086 & 0.770 & 0.920 & $0.524-1.612$ \\
\hline LUL & -0.188 & 0.288 & 0.429 & 0.513 & 0.828 & $0.471-456.000$ \\
\hline Histology & 0.335 & 0.287 & 1.362 & 0.243 & 1.399 & $0.796-2.457$ \\
\hline CADM2 mRNA & 0.437 & 0.196 & 4.989 & 0.026 & 1.548 & $1.055-2.270$ \\
\hline Extracranial lesions & -0.114 & 0.214 & 0.282 & 0.595 & 0.893 & $0.587-1.357$ \\
\hline Smoking & 0.318 & 0.191 & 2.781 & 0.095 & 1.375 & $0.946-1.999$ \\
\hline
\end{tabular}

NSCLC, non-small cell lung cancer; HR, hazard ratio; Cl, confidence interval; RUL, right upper lobe; RML, right middle lobe; RLL, right lower lobe; LUL, left upper lobe; CADM2, cell adhesion molecule 2.

Table 7 Multivariate survival analyses for overall survival according to the Cox regression model

\begin{tabular}{lcccccc}
\hline Variable & $\mathrm{B}$ & $\mathrm{SE}$ & Walds & $\mathrm{P}$ & $\mathrm{HR}$ & $95 \% \mathrm{Cl}$ \\
\hline Age (years) & 0.422 & 0.219 & 3.700 & 0.054 & 1.524 & $0.992-2.342$ \\
CADM2 mRNA & 0.399 & 0.197 & 4.109 & 0.043 & 1.490 & $1.013-2.191$ \\
\hline
\end{tabular}

SE, standard error; HR, hazard ratio; Cl, confidence interval; CADM2, cell adhesion molecule 2.

In conclusion, our study demonstrated that high CADM2 expression was associated with poor prognosis of NSCLC patients with brain metastases. CADM2 expression may represent a novel target for patients with brain metastasis that are poorly treated by conventional therapy. Improvements in screening, a more thorough understanding of tumor gene expression, and novel targets that impact actionable mutations represent important ways to reduce the mortality associated with NSCLC.

\section{Acknowledgments}

The authors appreciate the academic support from AME Lung Cancer Collaborative Group.

Funding: This study was supported by grants from the Guangdong Basic and Applied Basic Research Foundation (2019A1515011092, 2019A1515010026), Guangzhou
Science and Technology Program (202002030029, 202002030006).

\section{Footnote}

Reporting Checklist: The authors have completed the REMARK reporting checklist. Available at http://dx.doi. org/10.21037/jtd-21-307

Data Sharing Statement: Available at http://dx.doi. org/10.21037/jtd-21-307

Conflicts of Interest: All authors have completed the ICMJE uniform disclosure form (available at http://dx.doi. org/10.21037/jtd-21-307). Dr. AA reports personal fees from BMS, MSD, Astrazeneca, Pfizer, Roche, Boehringer, Ely-Lilly, outside the submitted work. The other authors 
have no conflicts of interest to declare.

Ethical Statement: The authors are accountable for all aspects of the work in ensuring that questions related to the accuracy or integrity of any part of the work are appropriately investigated and resolved. The study was approved by the local research ethics committee of Affiliated Cancer Hospital \& Institute of Guangzhou Medical University (No. 2020-57), and written informed consent was obtained from all subjects for sample collection and research use. All procedures performed in this study involving human participants were in accordance with the Declaration of Helsinki (as revised in 2013).

Open Access Statement: This is an Open Access article distributed in accordance with the Creative Commons Attribution-NonCommercial-NoDerivs 4.0 International License (CC BY-NC-ND 4.0), which permits the noncommercial replication and distribution of the article with the strict proviso that no changes or edits are made and the original work is properly cited (including links to both the formal publication through the relevant DOI and the license). See: https://creativecommons.org/licenses/by-nc-nd/4.0/.

\section{References}

1. Bray F, Ferlay J, Soerjomataram I, et al. Global cancer statistics 2018: GLOBOCAN estimates of incidence and mortality worldwide for 36 cancers in 185 countries. CA Cancer J Clin 2018;68:394-424.

2. Siegel R, Naishadham D, Jemal A. Cancer statistics, 2013. CA Cancer J Clin 2013;63:11-30.

3. Saad AG, Yeap BY, Thunnissen FBJM, et al. Immunohistochemical markers associated with brain metastases in patients with nonsmall cell lung carcinoma. Cancer 2008;113:2129-38.

4. Sperduto PW, Yang TJ, Beal K, et al. Estimating Survival in Patients with Lung Cancer and Brain Metastases: An Update of the Graded Prognostic Assessment for Lung Cancer Using Molecular Markers (Lung-molGPA). JAMA Oncol 2017;3:827-31.

5. Ceresoli GL, Reni M, Chiesa G, et al. Brain metastases in locally advanced nonsmall cell lung carcinoma after multimodality treatment: risk factors analysis. Cancer 2002;95:605-12.

6. Mujoomdar A, Austin J H M, Malhotra R, et al. Clinical predictors of metastatic disease to the brain from nonsmall cell lung carcinoma: primary tumor size, cell type, and lymph node metastases. Radiology 2007;242:882-8.

7. Wang SY, Ye X, Ou W, et al. Risk of cerebral metastases for postoperative locally advanced non-small-cell lung cancer. Lung Cancer 2009;64:238-43.

8. Won YW, Joo J, Yun T, et al. A nomogram to predict brain metastasis as the first relapse in curatively resected non-small cell lung cancer patients. Lung Cancer 2015;88:201-7.

9. Lee H, Jeong SH, Jeong BH, et al. Incidence of Brain Metastasis at the Initial Diagnosis of Lung Squamous Cell Carcinoma on the Basis of Stage, Excluding Brain Metastasis. J Thorac Oncol 2016;11:426-31.

10. Waqar SN, Waqar SH, Trinkaus K, et al. Brain Metastases at Presentation in Patients With Non-Small Cell Lung Cancer. Am J Clin Oncol 2018;41:36-40.

11. Waqar SN, Samson PP, Robinson CG, et al. Non-smallcell Lung Cancer With Brain Metastasis at Presentation. Clin Lung Cancer 2018;19:e373-9.

12. Breindel JL, Haskins JW, Cowell EP, et al. EGF receptor activates MET through MAPK to enhance non-small cell lung carcinoma invasion and brain metastasis. Cancer Res 2013;73:5053-65.

13. Li Q, Yang J, Yu Q, et al. Associations between singlenucleotide polymorphisms in the PI3K-PTEN-AKTmTOR pathway and increased risk of brain metastasis in patients with non-small cell lung cancer. Clin Cancer Res 2013;19:6252-60.

14. Kang HJ, Lim HJ, Park JS, et al. Comparison of clinical characteristics between patients with ALK-positive and EGFR-positive lung adenocarcinoma. Respir Med 2014;108:388-94.

15. Shin DY, Kim CH, Park S, et al. EGFR Mutation and Brain Metastasis in Pulmonary Adenocarcinomas. J Thorac Oncol 2014;9:195-9.

16. Grinberg-Rashi H, Ofek E, Perelman M, et al. The expression of three genes in primary non-small cell lung cancer is associated with metastatic spread to the brain. Clin Cancer Res 2009;15:1755-61.

17. Ge M, Zhuang Y, Zhou X, et al. High probability and frequency of EGFR mutations in non-small cell lung cancer with brain metastases. J Neurooncol 2017;135:413-8.

18. Chang WY, Wu YL, Su PL, et al. The impact of EGFR mutations on the incidence and survival of stages I to III NSCLC patients with subsequent brain metastasis. PLoS One 2018;13:e0192161.

19. Peters S, Camidge DR, Shaw AT, et al. Alectinib versus Crizotinib in Untreated ALK-Positive Non-Small-Cell Lung Cancer. N Engl J Med 2017;377:829-38.

20. Guérin A, Sasane M, Zhang J, et al. Brain metastases in 
patients with ALK+ non-small cell lung cancer: clinical symptoms, treatment patterns and economic burden. J Med Econ 2015;18:312-22.

21. Murakami Y. Involvement of a cell adhesion molecule, TSLC1/IGSF4, in human oncogenesis. Cancer Sci 2005;96:543-52.

22. van der Weyden L, Arends MJ, Rust AG, et al. Increased tumorigenesis associated with loss of the tumor suppressor gene Cadm1. Mol Cancer 2012;11:29.

23. Williams YN, Masuda M, Sakurai-Yageta M, et al. Cell adhesion and prostate tumor-suppressor activity of TSLL2/IGSF4C, an immunoglobulin superfamily molecule homologous to TSLC1/IGSF4. Oncogene 2006;25:1446-53.

24. Raveh S, Gavert N, Spiegel I, et al. The cell adhesion nectin-like molecules $(\mathrm{Necl}) 1$ and 4 suppress the growth and tumorigenic ability of colon cancer cells. J Cell Biochem 2009;108:326-36.

25. Nagata M, Sakurai-Yageta M, Yamada D, et al. Aberrations of a cell adhesion molecule CADM4 in renal clear cell carcinoma. Int J Cancer 2012;130:1329-37.

26. Dai L, Zhao J, Yin J, et al. Cell adhesion molecule 2 (CADM2) promotes brain metastasis by inducing epithelial-mesenchymal transition (EMT) in human nonsmall cell lung cancer. Ann Transl Med 2020;8:465.

27. Lee DS, Kim YS, Jung SL, et al. The relevance of serum carcinoembryonic antigen as an indicator of brain

Cite this article as: Dai L, Li YH, Liang YY, Zhao J, Chen G, Yin J, Postmus PE, Addeo A, Blasberg JD, Onesti CE, Liao ZW, Rao XG, Long HD. High expression of cell adhesion molecule 2 unfavorably impacts survival in non-small cell lung cancer patients with brain metastases. J Thorac Dis 2021;13(4):24372446. doi: $10.21037 /$ jtd-21-307 metastases detection in advanced non-small cell lung cancer. Tumour Biol 2012;33:1065-73.

28. Li D, Zhang Y, Zhang H, et al. CADM2, as a new target of miR-10b, promotes tumor metastasis through FAK/AKT pathway in hepatocellular carcinoma. J Exp Clin Cancer Res 2018;37:46.

29. Yang S, Yan HL, Tao QF, et al. Low CADM2 expression predicts high recurrence risk of hepatocellular carcinoma patients after hepatectomy. J Cancer Res Clin Oncol 2014;140:109-16.

30. Huang YX, Nie XG, Li GD, et al. Downregulation of microRNA-182 inhibits cell viability, invasion and angiogenesis in retinoblastoma through inhibition of the PI3K/AKT pathway and CADM2 upregulation. Int J Oncol 2018;53:2615-26.

31. Liu N, Yang C, Bai W, et al. CADM2 inhibits human glioma proliferation, migration and invasion. Oncol Rep 2019;41:2273-80.

32. Yang L, Zhao G, Wang F, et al. Hypoxia-Regulated miR146a Targets Cell Adhesion Molecule 2 to Promote Proliferation, Migration, and Invasion of Clear Cell Renal Cell Carcinoma. Cell Physiol Biochem 2018;49:920-31.

33. He W, Li X, Xu S, et al. Aberrant methylation and loss of CADM2 tumor suppressor expression is associated with human renal cell carcinoma tumor progression. Biochem Biophys Res Commun 2013;435:526-32. 